\section{A comprehensive review of adverse events associated with non-steroidal anti-inflammatory drug treatments in dogs in the UK}

\section{Jane Pigott ${ }^{1}$, Malcolm Cobb ${ }^{2}$, Declan O'Rourke ${ }^{2}$}

Gurrane, Dromagh, Mallow, Co.Cork, Ireland

School of Veterinary Medicine and Science, University of Nottingham,

Sutton Bonnington, Leicestershire LE12 5RD, UK

Non-steroidal anti-inflammatory drugs (NSAIDs) are used in the management of painful and inflammatory conditions in dogs. The use of these agents is not without risk, as adverse events are observed. This study aims to evaluate the nature and frequency with which reactions occur following the use of carprofen and meloxicam.

This study used data extracted from the suspected adverse reaction surveillance scheme (SARSS) database of the Veterinary Medicines Directorate (April 1992-October 2011). The data for each active was separated on the basis of whether the reported use was authorised or unauthorised. Using the Veterinary Dictionary for Drug Related affairs, the clinical sign provided in the original information was converted into the 'preferred term', and allocated the appropriate 'system organ class' (SOC).

The total number of signs reported in each SOC was calculated, and the frequency with which each system was reported determined. A breakdown of the signs reported for the 5 most prevalent SOCs was then completed. The proportion of signs in each SOC category associated with authorised use was compared for carprofen and meloxicam. A Chi-squared test was used to compare the frequency with which each reaction occurred for each of the two actives (GraphPad InStat 3, produced by GraphPad Software). Significance was set at $P<0.05$.

The data of this study reported 676 cases of adverse reactions to carprofen and 567 cases of adverse reactions to meloxicam since the actives were authorised in the UK. The most common adverse reactions reported for authorised use of carprofen were systemic disorders (37.06\%), digestive tract disorders (24.43\%), neurological disorders (7.36\%) and blood and lymphatic system disorders (5.87\%). The most common adverse reactions reported for authorised use of meloxicam were digestive tract disorders $(37.76 \%)$, systemic disorders (32.74\%), neurological disorders (5.69\%), and blood and lymphatic system disorders (3.99\%).

Meloxicam was found to cause more digestive tract disorders, cardio-vascular system disorders and reproductive tract disorders than carprofen, whereas carprofen caused more application site disorders, hepato-biliary disorders, systemic disorders, blood and lymphatic system disorders, ear and labyrinth disorders, neurological disorders and musculoskeletal disorders than meloxicam.

Systemic and digestive tract disorders were the most commonly reported reactions following unauthorised use of both actives.

This study provides a comprehensive evaluation of carprofen and meloxicam related adverse reactions in the dog in the UK.

\section{The novel use of fluroscopy for guided insertion of thoracostomy tubes in dogs}

\section{Tonje Trinterud, Jackie Demetriou}

Dick White Referrals, Six Mile Bottom, UK

\section{INTRODUCTION}

Thoracostomy tubes are used for removal of intrathoracic accumulation of fluid or air. The current standard for insertion of thoracostomy tubes is 'blind' placement technique. Not being able to visualize the position of the tube during placement may lead to iatrogentic damage to the intrathoracic structures or failure of drainage.

\section{AlM}

Prospectively compare fluoroscopically guided thoracostomy tube placement with 'blinded' tube placement

\section{MATERIAL AND METHODS}

Dogs were divided into two groups, 'blinded placement' (BP) or fluoroscopically guided placement (FGP).

Wide bore thoracostomy tubes were used in both groups.
Dogs with pleural effusion were assigned to BP, while FGP was performed in dogs undergoing lateral thoracotomy.

Tube position, minor/major adjustments, tube insertion time and time to satisfactory tube placement documented on radiographs and resulting in drainage were recorded.

Minor adjustments were defined as adjustments performed without removing the tube from thorax. Major adjustments were defined as adjustments requiring removal and re-insertion of the tube in the thorax.

\section{RESULTS}

Twelve adult dogs (six in each group) were included.

Tube position, BP: $2 / 6$ tubes entered thorax through 6 th intercostal space (IC), 4/6 through 7th IC, cranial tip of tube ended at 1 st rib in $2 / 6$, 3rd rib $2 / 6$ and 4 th rib $2 / 6$ tubes, the tube tip was positioned in ventral third of thorax in $5 / 6$ and in middle third in $1 / 6$ dogs

Tube position, FGP: 2/6 tubes entered thorax through 6th intercostal space (IC), 4/6 through 7th IC, cranial tip of tube ended at 2 nd rib in $6 / 6$ tubes, the tube tip was positioned in ventral third of thorax in $3 / 6$ and in middle third in $3 / 6$ dogs

Minor adjustments: BP: 2/6, FGP: 3/6

Major adjustments: BP: 2/6, FGP: 0/6

Mean placement time: BP: 2 min $30 \mathrm{sec}, \mathrm{FGP}: 1 \mathrm{~min} 50 \mathrm{sec}$

Mean time to documented satisfactory placement and drainage: BP: $27 \mathrm{~min} 30 \mathrm{sec}$, FGP: $1 \mathrm{~min} 50 \mathrm{sec}$ 


\section{Congress 2015}

\section{DISCUSSION AND CONCLUSION}

Thoracic tube insertion, both 'blinded' and fluoroscopically guided are relatively fast to perform, and result in accurate placement with satisfactory drainage. Predicting the tube positioning after thoracic entry may be difficult. By using fluoroscopy the tube can be visually guided through the thoracic cavity and minor adjustments may be performed during insertion. The overall procedure time may be reduced with fluoroscopy as postoperative radiography is unnecessary.

\section{Prognostic factors in acute-onset feline congestive heart failure: a retrospective study of 34 cases}

\section{Lamprini Kalogianni', loannis Kavarnos ${ }^{1}$, Despina Alatza', Soubasis Nektarios', Christos Koutinas', Michail Patsikas', Dimitris Kasabalis ${ }^{3}$}

Companion Animal Clinic, School of Veterinary Medicine, Faculty of Health Sciences, Aristotle University of Thessaloniki, Thessaloniki, Greece

Department of Diagnostic Imaging, School of Veterinary Medicine, Faculty of Health Sciences, Aristotle University of Thessaloniki, Thessaloniki, Greece

Clinic of Medicine, Faculty of Veterinary Medicine, University of Thessaly, Karditsa, Greece

\section{INTRODUCTION}

Feline congestive heart failure (CHF) is the result of underlying cardiomyopathy. The aim of this retrospective study was to evaluate physical and laboratory findings in admission as prognostic factors in cats with CHF.

\section{MATERIALS AND METHODS}

Medical records from 1996 to 2011 were reviewed retrospectively for cats with CHF (presence of pulmonary edema, thoracic effusion or ascites) and a confirmed diagnosis of cardiomyopathy. Incomplete records and any treatment prior to admission were the exclusion criteria. The main physical examination and laboratory findings were recorded and their effect on treatment outcome and survival was evaluated. Correlations were tested by chi-square test and continuous data were compared using t-test and ANOVA tests, or Kruskal-Wallis and Mann-Whitney. The statistical significance level was defined at 0.05 .

\section{RESULTS}

A total of 34 cats were eligible for the study. Breeds included the Domestic Shorthair $(44.1 \%, 15 / 34)$, Siamese $(41.2 \%$, 14/34), Domestic Longhair (8.8\%, 3/34) and Angora (5.9\%, $2 / 34) ; 61.8 \%$ were males (21/34) and $38.2 \%$ were females $(13 / 34)$. Most affected cats were above the age of 11 years $(35.3 \%, 12 / 34)$. Conclusive diagnosis included hypertrophic $(67.6 \%, 23 / 34)$, dilated $(26.5 \%, 9 / 34)$, unclassified $(2.9 \%$, $1 / 34)$, and restrictive cardiomyopathy $(2.9 \%, 1 / 34)$. Common physical examination findings were dyspnoea $(55.9 \%, 19 / 34)$, hypothermia $(50 \%, 16 / 32)$, tachycardia $(48.5 \%, 16 / 33$ cats), weak peripheral pulse $(34.4 \%, 11 / 32)$, gallop rhythm $(29 \%$, $9 / 31)$, systolic murmur $(23.5 \%, 8 / 34)$ and bradycardia $(15.2 \%$, $5 / 34$ ). Auscultatory findings (rhonchi) were present in $11.8 \%$ (4/34), ascites in $32.4 \%(11 / 34)$ and pleural effusion in $94.1 \%$ $(32 / 34)$ of cases. Laboratory findings included anemia $(21.9 \%$, $7 / 32$ ) azotemia (elevation in blood urea nitrogen in $65.4 \%$, $17 / 26$ and in creatinine concentrations in 44.4\%, 12/27), hyponatremia $(20 \%, 4 / 20)$ hypokalemia $(15.4 \%, 4 / 26)$ and hyperkalemia $(11.5 \%, 3 / 26)$. From a total of 34 cats, $67.6 \%$ of cats $(23 / 34)$ were hospitalized, 13 of which survived to be discharged and relapsed in 2-270 days (median 10 days). Hypertrophic cardiomyopathy was prevalent in cats older than 11 years. There was a positive association between tachycardia and discharge. Only bradycardia had a statistically significant negative effect on survival.

\section{CLINICAL IMPLICATIONS}

A relatively uniform clinical presentation was observed in cats with CHF. Congestive heart failure was noted increasingly in aged cats with hypertrophic cardiomyopathy. Even though hypothermia, respiratory rate and azotemia have been reported as negative prognostic factors in cats, in this study bradycardia was the only clinical finding to negatively affect the short-term prognosis.
Quantifying the role of progestogen and glucocorticoid exposure in the epidemiology of feline diabetes mellitus in Romania

\author{
Madalina Rosca', Gheorghe Solcan', \\ David C. Brodbelt ${ }^{2}$, Stijn J. M. Niessen ${ }^{3}$
}

University of Agricultural Sciences and Veterinary Medicine, Faculty of Veterinary Medicine, Small Animal Internal Medicine Department, Iasi, Romania

Royal Veterinary College, Department of Production and Population Health, London, UK

Royal Veterinary College, Department of Clinical Sciences and Services, London, UK

\section{BACKGROUND}

Progestogen and glucocorticoid administration have been documented as a risk factor for the development of feline diabetes mellitus (DM) in a handful of studies. Despite this, they remain popular among veterinarians. Additiona information regarding their exact diabetogenic risk could aid 